Supplement of Atmos. Chem. Phys. Discuss., 15, 26959-26995, 2015

http://www.atmos-chem-phys-discuss.net/15/26959/2015/

doi:10.5194/acpd-15-26959-2015-supplement

(C) Author(s) 2015. CC Attribution 3.0 License.

(c) (i)

\title{
High-resolution ammonia emissions inventories in China from 1980-2012
}

\section{Y. Kang et al.}

Correspondence to: Y. Song (songyu@pku.edu.cn)

The copyright of individual parts of the supplement might differ from the CC-BY 3.0 licence. 
Table S1. Parameters used in estimates of annual TAN excretion per animal for each livestock class.

\begin{tabular}{|c|c|c|c|c|c|c|}
\hline \multirow{2}{*}{$\begin{array}{l}\text { Livestock } \\
\text { class }\end{array}$} & \multirow{2}{*}{$\begin{array}{l}\text { Period }^{\mathrm{a}} \\
\text { (day) }\end{array}$} & \multicolumn{2}{|c|}{$\begin{array}{c}\text { Excrement }^{\mathrm{b}} \\
\text { (Kg/day/ capita) }\end{array}$} & \multicolumn{2}{|c|}{$\begin{array}{c}\text { Nitrogen contents } \\
(\%)\end{array}$} & \multirow{2}{*}{$\begin{array}{c}\text { TAN } \\
\text { content } \\
(\%)\end{array}$} \\
\hline & & Urine & Faece & Urine & Faece & \\
\hline Beef <1year & 365 & 5.00 & 7.0 & 0.90 & 0.38 & 60 \\
\hline Beef $>1$ year & 365 & 10.00 & 20.0 & 0.90 & 0.38 & 60 \\
\hline $\begin{array}{l}\text { Dairy } \\
\text { cows }<1 \text { year }\end{array}$ & 365 & 5.00 & 7.0 & 0.90 & 0.38 & 60 \\
\hline $\begin{array}{l}\text { Dairy } \\
\text { cows }>1 \text { year }\end{array}$ & 365 & 19.00 & 40.0 & 0.90 & 0.38 & 60 \\
\hline Goat<1year & 365 & 0.66 & 1.5 & 1.35 & 0.75 & 60 \\
\hline Goat>1year & 365 & 0.75 & 2.6 & 1.35 & 0.75 & 50 \\
\hline Sheep <1year & 365 & 0.66 & 1.5 & 1.35 & 0.75 & 60 \\
\hline Sheep > 1year & 365 & 0.75 & 2.6 & 1.35 & 0.75 & 50 \\
\hline Sow & 365 & 5.70 & 2.1 & 0.40 & 0.34 & 70 \\
\hline Weaner & 75 & 1.20 & 0.5 & 0.40 & 0.34 & 70 \\
\hline Fattening pig & 75 & 3.20 & 1.5 & 0.40 & 0.34 & 70 \\
\hline Horse & 365 & 6.50 & 15.0 & 1.40 & 0.20 & 60 \\
\hline Donkey & 365 & 6.50 & 15.0 & 1.40 & 0.20 & 60 \\
\hline Mule & 365 & 6.50 & 15.0 & 1.40 & 0.20 & 60 \\
\hline Camel & 365 & 6.50 & 15.0 & 1.40 & 0.20 & 60 \\
\hline Laying hen & 365 & - & 0.12 & - & 1.63 & 70 \\
\hline Laying duck & 365 & - & 0.13 & - & 1.10 & 70 \\
\hline Laying goose & 365 & - & 0.13 & - & 0.55 & 70 \\
\hline Broilers & 50 & - & 0.09 & - & 1.63 & 70 \\
\hline Meat duck & 55 & - & 0.10 & - & 1.10 & 70 \\
\hline Meat goose & 70 & - & 0.10 & - & 0.55 & 70 \\
\hline
\end{tabular}

${ }^{a}$ Values are derived from CAU (1997); Zhou et al. (2010).

${ }^{\mathrm{b}}$ Values are derived from CAU (1997); MEPPRC (2001); Wang et al. (2009)

${ }^{\mathrm{c}}$ Values are derived from CAU (1978).

${ }^{\mathrm{d}}$ Values are derived from Webb and Misselbrook (2004); EEA (2013)

Table S2. The defined threshold of intensive rearing system for different livestock categories (unit is capita)*.

\begin{tabular}{cccccc}
\hline Beef & Dairy cow & Goat/Sheep & Pig & $\begin{array}{c}\text { Laying } \\
\text { hen }\end{array}$ & Broiler \\
\hline 50 & 100 & 100 & 500 & 2000 & 50000 \\
\hline
\end{tabular}

* The values are derived from EOCAIY (2007); when the values exceed above threshold, they are defined as intensive system. 
Table S3. Interannual emissions (Gg) of typical livestock categories from 1980 to 2012.

\begin{tabular}{|c|c|c|c|c|c|c|c|c|c|c|c|c|}
\hline & Beef & $\begin{array}{l}\text { Dairy } \\
\text { cows }\end{array}$ & Goat & Sheep & Pigs & Horse & Donkey & Mule & $\begin{array}{l}\text { Laying } \\
\text { hen }\end{array}$ & $\begin{array}{l}\text { Laying } \\
\text { duck }\end{array}$ & Broilers & $\begin{array}{l}\text { Meat } \\
\text { duck }\end{array}$ \\
\hline 1980 & 1327 & 54 & 286 & 398 & 284 & 183 & 129 & 74 & 73 & 24 & 20 & 3 \\
\hline 1981 & 1369 & 35 & 279 & 406 & 275 & 189 & 130 & 74 & 79 & 26 & 22 & 3 \\
\hline 1982 & 1403 & 57 & 267 & 395 & 282 & 191 & 179 & 85 & 94 & 30 & 25 & 3 \\
\hline 1983 & 1437 & 58 & 242 & 367 & 285 & 191 & 179 & 85 & 114 & 36 & 31 & 4 \\
\hline 1984 & 1505 & 60 & 226 & 353 & 299 & 191 & 179 & 85 & 133 & 42 & 36 & 5 \\
\hline 1985 & 1592 & 64 & 220 & 349 & 324 & 191 & 179 & 85 & 158 & 49 & 43 & 5 \\
\hline 1986 & 1680 & 67 & 241 & 367 & 341 & 189 & 184 & 88 & 154 & 50 & 42 & 6 \\
\hline 1987 & 1725 & 75 & 275 & 379 & 339 & 185 & 187 & 90 & 160 & 51 & 44 & 6 \\
\hline 1988 & 1779 & 77 & 321 & 407 & 358 & 182 & 190 & 92 & 182 & 55 & 49 & 6 \\
\hline 1989 & 1824 & 84 & 345 & 418 & 374 & 183 & 191 & 92 & 180 & 56 & 48 & 6 \\
\hline 1990 & 1850 & 98 & 362 & 394 & 398 & 168 & 191 & 93 & 192 & 60 & 52 & 7 \\
\hline 1991 & 1880 & 99 & 353 & 390 & 411 & 175 & 192 & 96 & 172 & 68 & 67 & 8 \\
\hline 1992 & 1925 & 101 & 356 & 386 & 433 & 174 & 189 & 96 & 191 & 77 & 76 & 9 \\
\hline 1993 & 2014 & 104 & 383 & 393 & 459 & 173 & 191 & 98 & 243 & 95 & 95 & 11 \\
\hline 1994 & 2183 & 114 & 436 & 417 & 499 & 162 & 188 & 95 & 327 & 119 & 120 & 13 \\
\hline 1995 & 2328 & 126 & 519 & 461 & 559 & 174 & 185 & 92 & 417 & 146 & 147 & 16 \\
\hline 1996 & 2456 & 134 & 588 & 481 & 498 & 70 & 184 & 92 & 479 & 165 & 167 & 18 \\
\hline 1997 & 2036 & 119 & 457 & 447 & 515 & 154 & 167 & 83 & 502 & 177 & 178 & 20 \\
\hline 1998 & 2170 & 121 & 512 & 474 & 571 & 154 & 167 & 83 & 476 & 159 & 156 & 18 \\
\hline 1999 & 2214 & 125 & 506 & 474 & 587 & 153 & 159 & 75 & 485 & 163 & 165 & 18 \\
\hline 2000 & 2230 & 150 & 541 & 486 & 634 & 153 & 159 & 75 & 548 & 179 & 179 & 20 \\
\hline 2001 & 2220 & 164 & 550 & 486 & 647 & 145 & 153 & 75 & 562 & 184 & 184 & 20 \\
\hline 2002 & 2250 & 204 & 575 & 503 & 666 & 142 & 148 & 72 & 577 & 190 & 188 & 21 \\
\hline 2003 & 2278 & 250 & 635 & 570 & 679 & 139 & 143 & 68 & 618 & 204 & 200 & 23 \\
\hline 2004 & 2292 & 308 & 665 & 607 & 697 & 134 & 138 & 65 & 638 & 209 & 201 & 23 \\
\hline 2005 & 2325 & 321 & 673 & 622 & 739 & 131 & 135 & 63 & 682 & 224 & 218 & 25 \\
\hline 2006 & 2076 & 306 & 661 & 609 & 741 & 112 & 128 & 60 & 684 & 229 & 223 & 26 \\
\hline 2007 & 1680 & 338 & 494 & 513 & 618 & 125 & 121 & 52 & 579 & 212 & 215 & 24 \\
\hline 2008 & 1663 & 340 & 522 & 464 & 661 & 121 & 118 & 52 & 597 & 224 & 227 & 26 \\
\hline 2009 & 1665 & 336 & 509 & 482 & 804 & 121 & 114 & 61 & 608 & 232 & 234 & 27 \\
\hline 2010 & 1657 & 378 & 475 & 491 & 671 & 120 & 113 & 49 & 619 & 240 & 240 & 28 \\
\hline 2011 & 1536 & 371 & 465 & 484 & 662 & 119 & 114 & 46 & 637 & 247 & 246 & 29 \\
\hline 2012 & 1511 & 388 & 388 & 401 & 349 & 113 & 112 & 44 & 684 & 262 & 260 & 31 \\
\hline
\end{tabular}




\section{Figure captions}

Figure S1. Annual populations of major livestock animals (cattle (a), sheep (b), pig (c), poultry (d)) in China from 1980 to 2012 (unit: million head); the values are derived from EOCAY (1981-2013) and EOCAIY (1999-2013).

Figure S2. Annual production of synthetic fertilizers (categorized as urea, ABC and others (AN, AS and others)) from 1980 to 2012 in China; the values are derived from Zhang et al. (2012). 

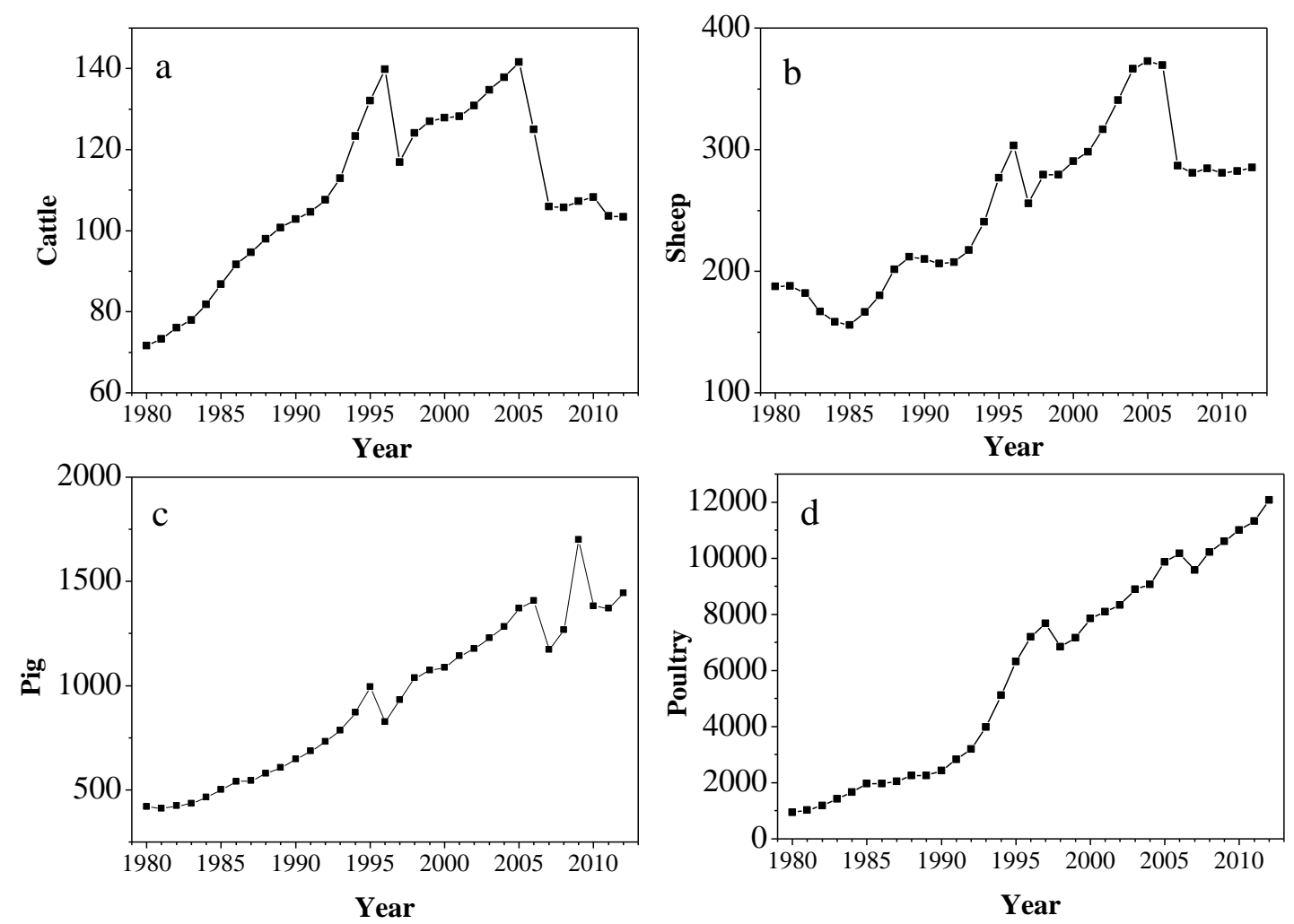

Figure S1. Annual populations of major livestock animals (cattle (a), sheep (b), pig (c), poultry (d)) in China from 1980 to 2012 (unit: million capital); the values are derived from EOCAY (1981-2013) and EOCAIY (1999-2013). 


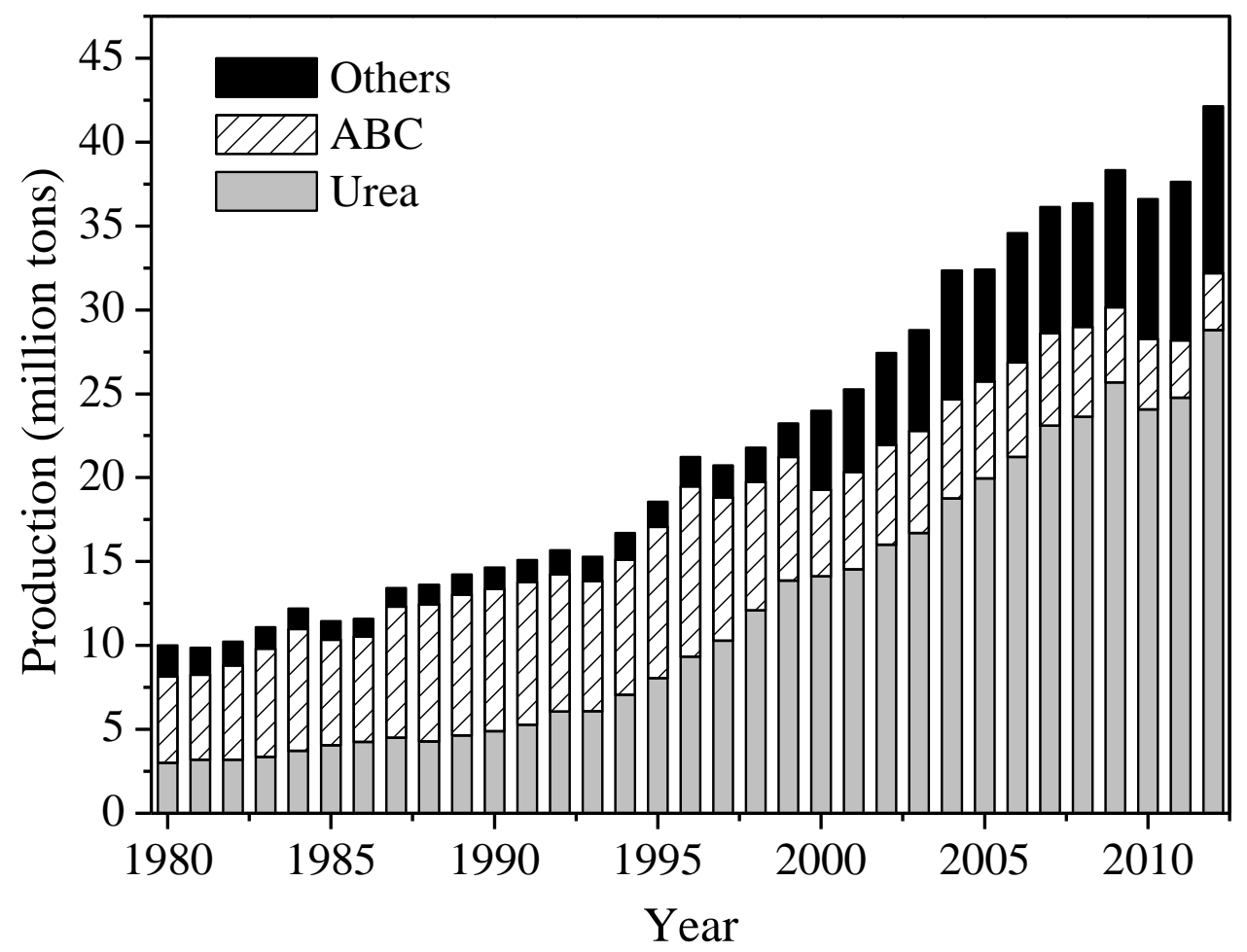

Figure S2. Annual production of synthetic fertilizers (categorized as urea, ABC and others (AN, AS and others)) from 1980 to 2012 in China; the values are derived from Zhang et al. (2012). 


\section{References:}

China Agricultural University (CAU): Fertilizer Manual [in Chinese], China Agriculture Press, Beijing, 1978.

China Agricultural University (CAU): Coprology of domestic animals [in Chinese], Shanghai Jiaotong University Press, Shanghai, 1997.

European Environment Agency (EEA): EMEP/EEA air pollutant emission inventory guidebook 2013, European Environment Agency, 2013.

Editorial Office of China Animal Industry Yearbook (EOCAIY): China Animal Industry Yearbook 1999-2013 [in Chinese], China Agriculture Press, Beijing, 1999-2013.

Editorial Office of China Animal Industry Yearbook (EOCAIY): China Animal Industry Yearbook 2007 [in Chinese], China Agriculture Press, Beijing, 2007.

Editorial Office of China Agriculture Yearbook (EOCAY): China Agriculture Yearbook 1981-2013 [in Chinese] China Agriculture Press, Beijing, 1981-2013.

Ministry of Environmental Protection of People's Republic of China (MEPPRC): The national large-scale livestock farming systems pollution situation investigation technical reports [in Chinese], Ministry of Environmental Protection of People's Republic of China, Beijing, 59pp, 2001.

Wang, K., Liu, J., Chen, X., Dai, X., and Feng, S.: Pollutant production and discharge from livestock and poultry industries and land carrying capacity in Zhejiang Province [in Chinese], Chinese Journal of Applied Ecology, 20, 3043-6048., 2009.

Webb, J., and Misselbrook, T. H.: A mass-flow model of ammonia emissions from UK livestock production, Atmos. Environ., 38, 2163-2176, doi: 10.1016/j.atmosenv.2004.01.023, 2004.

Zhang, W. F., Zhang, F. S., LI, Y. X., Huang, G. Q., and Wu, L.: China Fertilizer Development Report [in Chinese]. China Agricultural University Press, 2012.

Zhou, K., Lei, Z., Wang, Z., Shi, J., and Peng, Z.: Estimation of annual total livestock/poultry excrement in Henan Province [in Chinese], Chinese Journal of Eco-Agriculture, 18, 1060-1065, 2010. 